\title{
Radiation Detector Characterization at APO While Stacking pbars in 1999
}

The Main Injector provided beam for pbar stacking for the first time in 1999 over the period $12 / 20$ to $12 / 21$. The purpose of this memo is to record some observations on the response of various radiation detectors as a function of beam on the pbar target. The detectors include a Scarecrow in the APO Vault, a Chipmunk just upstream of the APO vault, and a Chipmunk in the water cage adjacent to the Pulsed Magnet pump skid in the water systems cage. In addition, there are air monitors, one sampling in the PreVault enclosure and one sampling at the exhaust stack at the upstream end of the PreTarget enciosure.

All data was collected by the ACNET system Lumberjack data logger. Beam intensity data was summed over consecutive 10 minute periods and normalized to an hourly intensity. The Chipmunk, Scarecrow, and Air Monitor data are based 10 minute averages taken over periods which coincide with normalized beam intensity.

In Figure 1, the relationship of the Chipmunks and Scarecrow are shown as a function of beam intensity. These detector responses appear to be linear and have the following slopes:
US Chipmunk
2.02E-15 mrem/p
Vault Scarecrow
$1.64 \mathrm{E}-14 \mathrm{mrem} / \mathrm{p}$
Water Cage Chipmunk
$1.92 \mathrm{E}-14 \mathrm{mrem} / \mathrm{p}$

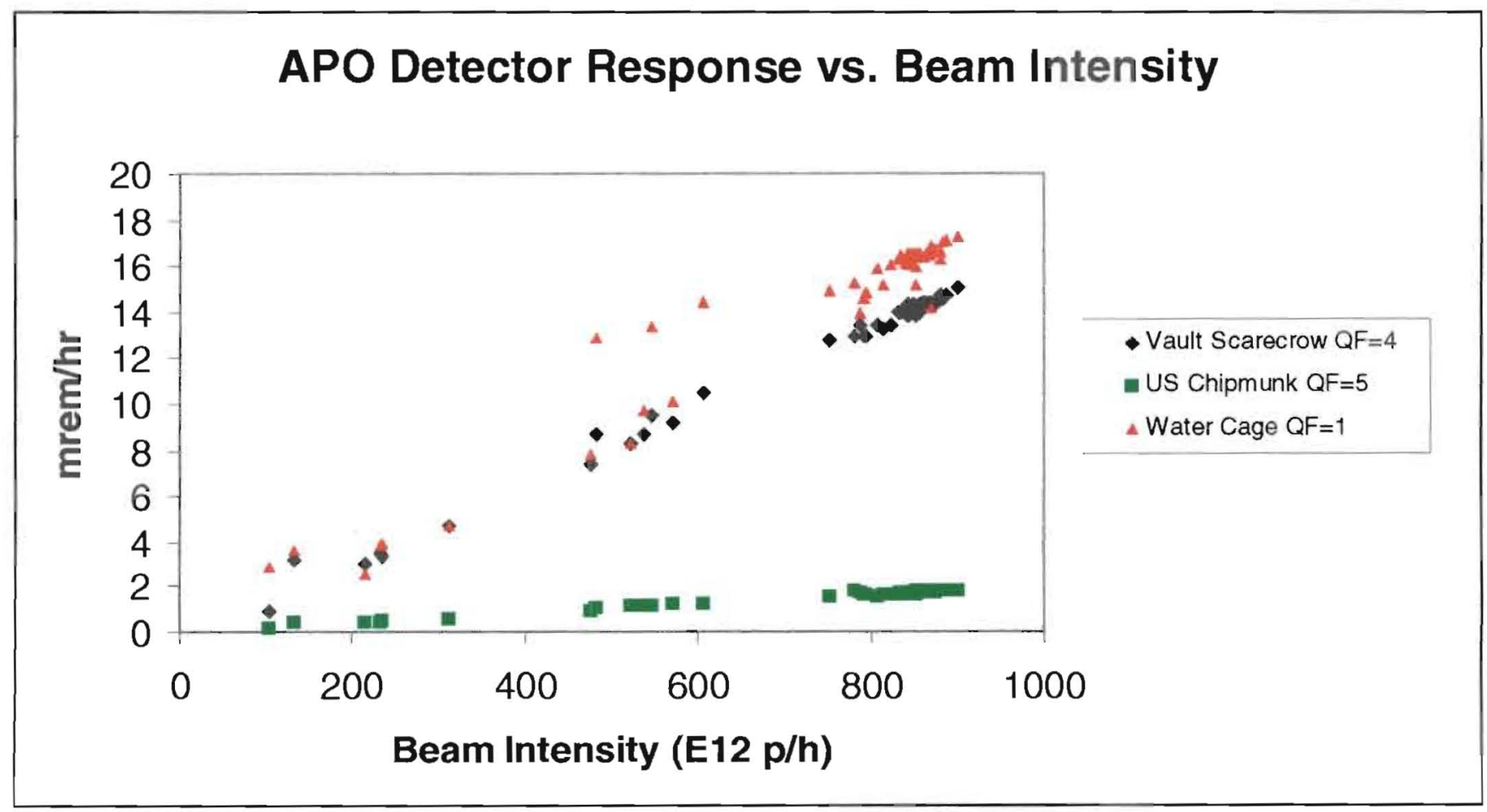

Figure 1 
The response of Chipmunks and Scarecrows to prompt radiation is usually direct and linear with beam intensity for any given tuning condition. Air monitor response is direct and linear with radioactivity in the air being sampled but because of the time delay between when the air is activated and when it is sampled, it is not typically linear and direct with beam intensity. It is believed that air temperature changes, air pressure variation, and ventilation controls weaken the relationship between air monitor response and beam intensity. Figure 2 shows air monitor response as a function of beam intensity.

\section{APO Air Monitor Response vs. Beam Intensity on target}
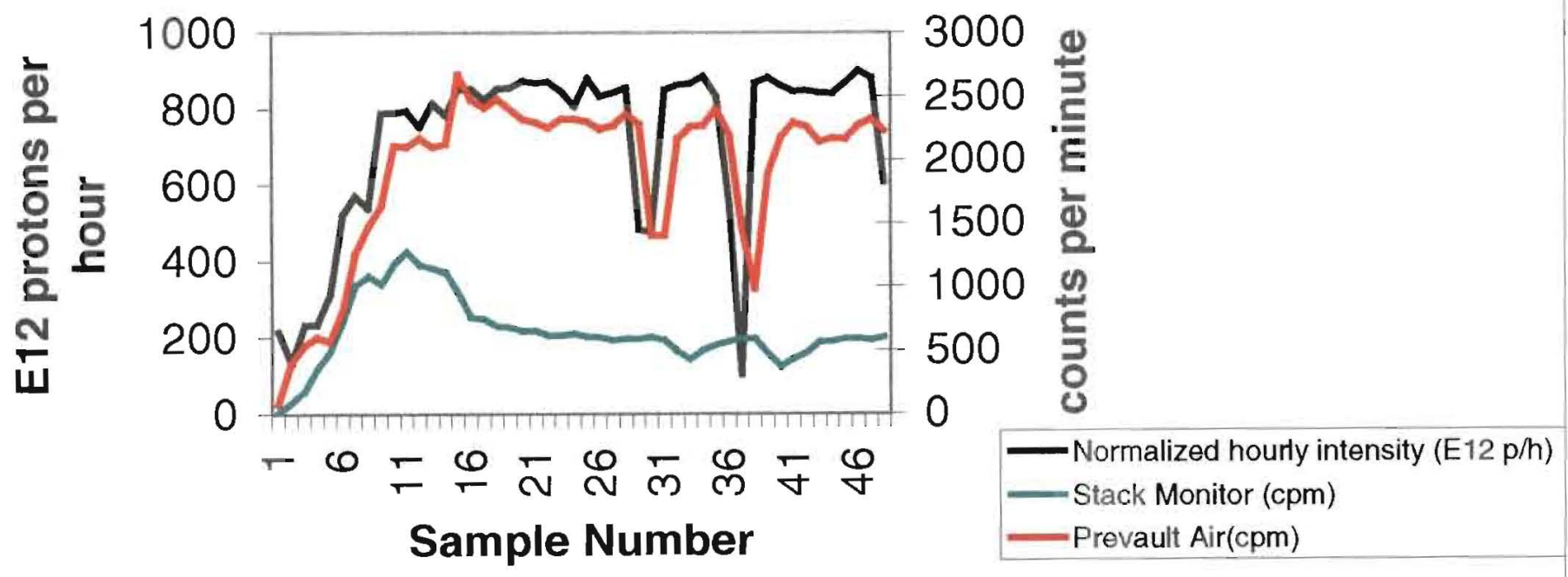

Air monitor samples based on 10 minute average

Beam intensity is integral of intensity over 10 minutes nomalized to one hour

Figure 2

Air monitor response does appear to be linear during steady state operation. Over the period $12 / 20$ to $12 / 21$, the following relationships were found:

$$
\begin{array}{ll}
\text { PreVault Air Monitor } & 2.58 \mathrm{E}-12 \mathrm{cpm} / \mathrm{p} / \mathrm{h} \\
\text { Stack Monitor } & 6.66 \mathrm{E}-13 \mathrm{cpm} / \mathrm{p} / \mathrm{h}
\end{array}
$$

Based upon these measurements, the upper limit of Chipmunk, Scarecrow, and Air Monitor response can be predicted for a given beam intensity limit. For the foreseeable future, the beam intensity limit for phar is intended to be $1.2 \mathrm{E} 16 \mathrm{p} / \mathrm{h}$. For steady state operation at the limiting beam intensity, Chipmunk, Scarecrow, and Air Monitor response is predicted to reach: 
Pbar Note 628, Revision 1

Detector Characterization at APO for First Beam to pbar in 1999

A. F. Leveling $2 / 9 / 00$

$\begin{array}{ll}\text { PreVault Air Monitor } & 31,000 \mathrm{cpm} \\ \text { Stack Monitor } & 8,000 \mathrm{cpm} \\ \text { US Chipmunk } & 24 \mathrm{mrem} / \mathrm{h} \\ \text { Vault Scarecrow } & 200 \mathrm{mrem} / \mathrm{h} \\ \text { Water Cage Chipmunk } & 230 \mathrm{mrem} / \mathrm{h}\end{array}$

The Water Cage Chipmunk was initially positioned about 2 feet from the Pulsed Magnet Water System and about 8 feet from the APO Dump Water System over the period 12/20 to $12 / 21$ when the values listed above were determined. The Water Cage Chipmunk was moved in January 2000 to a position directly adjacent to the Dump Water System to obtain dose information near to it since it was thought to be the most radioactive system. The resulting dose per proton for that position in a 1/11/00 measurement was 8.5E-14 $\mathrm{mrem} / \mathrm{p}$. To check reproducibility of the running condition with the December 1999 measurement, the Vault Scarecrow and US Chipmunk dose per proton values were rechecked and found to be $1.7 \mathrm{E}-14$ and $2.03 \mathrm{E}-15 \mathrm{mrem} / \mathrm{p}$, respectively. These values agree very well with those found in the December 1999 measurement. For steady state operation at the limiting beam intensity, the Chipmunk response at the APO Dump Water System is expected to reach:

$$
\text { APO Dump Water System } 1024 \mathrm{mrem} / \mathrm{h}
$$

A follow up measurement was made over the period $2 / 5$ to $2 / 6 / 00$ to understand the response of the upstream detector during stacking. An array of three additional chipmunks was set out at 4 foot intervals upstream of the existing interlocked chipmunk. Figure 3 shows a scale elevation view of the region along with the detector array and the resulting dose equivalent rate distribution. The shape of the loss pattern suggests that the source of the radiation field is the Vault enclosure and not the PreVault enclosure. Some additional measurements will be made with Chipmunks during the next stacking period to determine the dose rate distribution across the beam line. A set of TLD badges will be used to determine the contribution due to photons and neutrons. Based on this information, we can plan to install some additional shielding to reduce the dose rates in the region to more acceptable levels.

Finally, we will revisit the shielding in the PreVault region below the building to determine if further protective measures need to be considered.

Digital photographs of the water cage are included in this document. 
Pbar Note 628, Revision 1

Detector Characterization at APO for First Beam to pbar in 1999

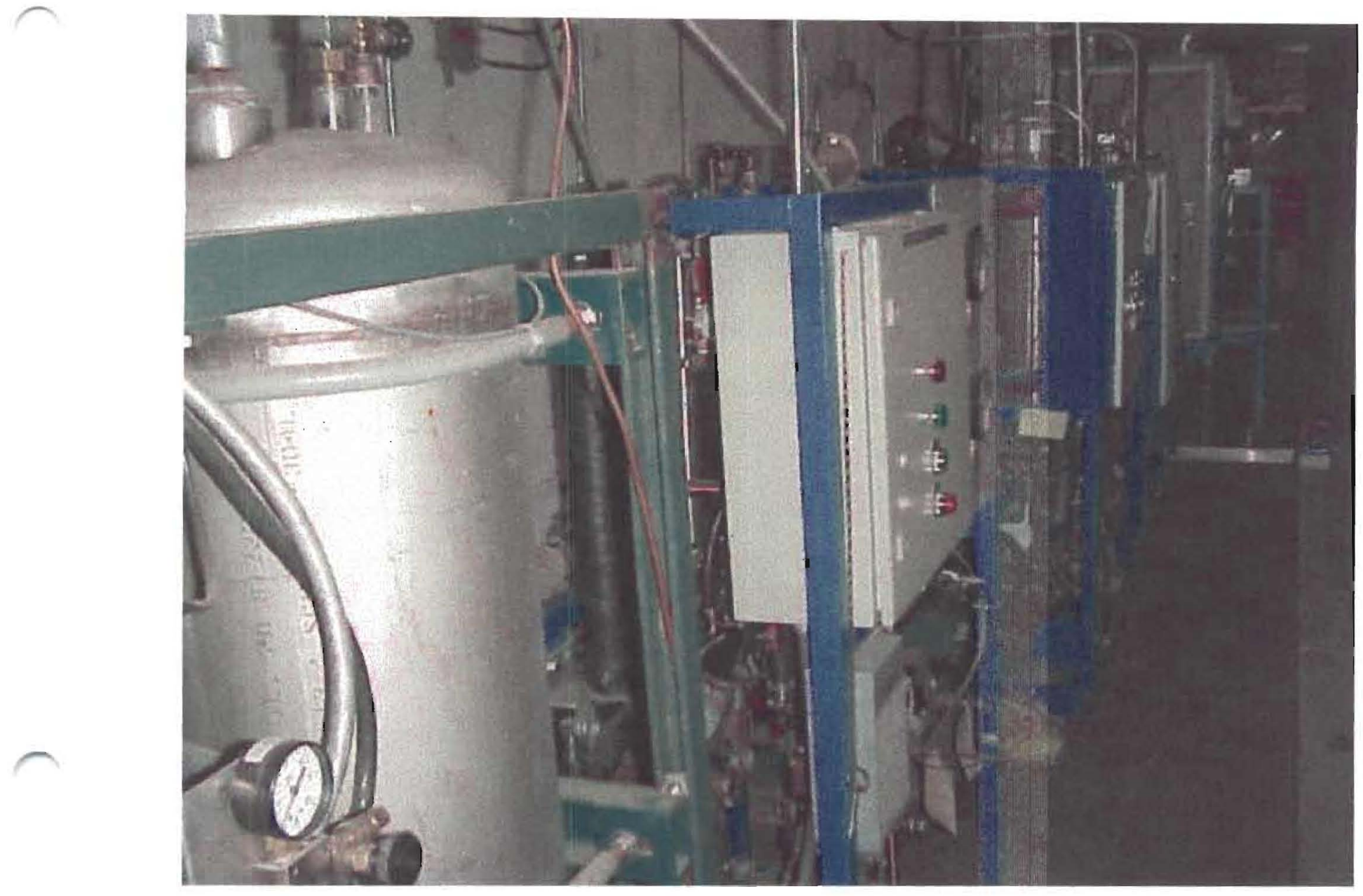

Proton Lens Cooling System (partial), Pulsed Magnet Cooling System, Dump Cooling System, and Lens Cooling System (left to right)
A. F. Leveling

$2 / 9 / 00$ 
Pbar Note 628, Revision 1

Detector Characterization at APO for First Beam to pbar in 1999
A. F. Leveling $2 / 9 / 00$

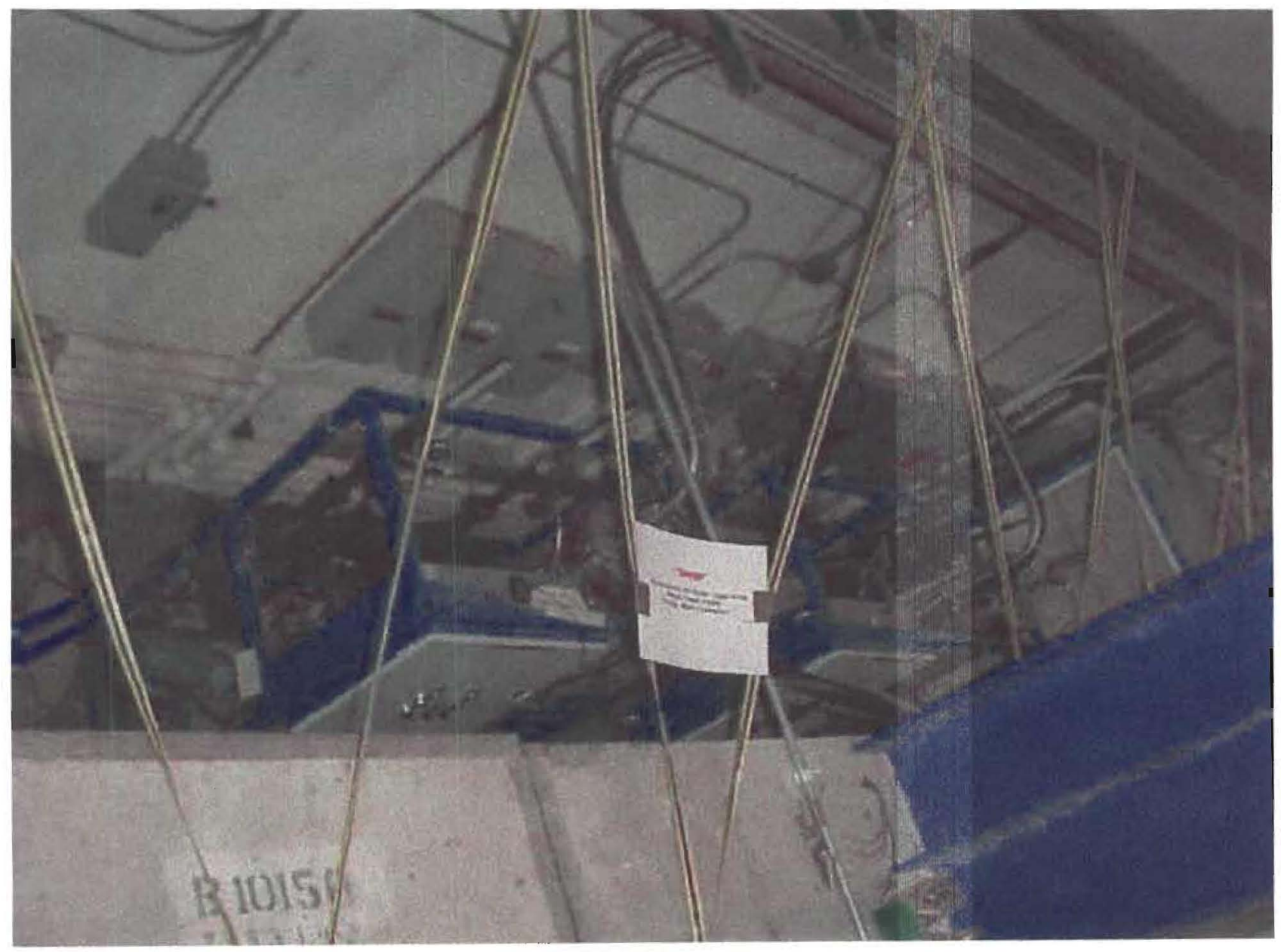

APO Dump Cooling System (left) and Lithium Lens Cooling System 
Pbar Note 628, Revision 1

Detector Characterization at APO for First Beam to pbar in 1999
A. F. Leveling $2 / 9 / 00$

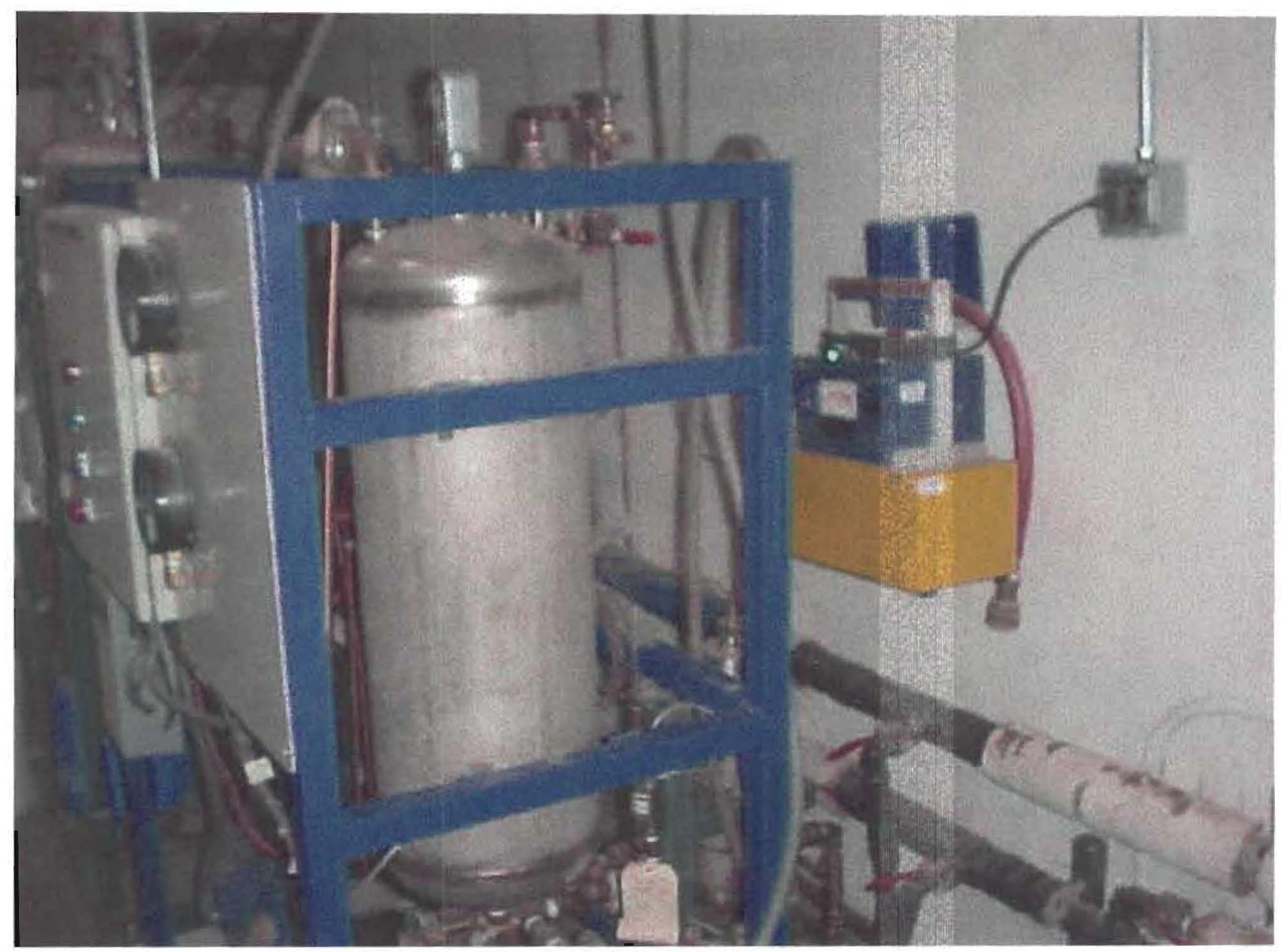

Pulsed Magnet Water Cooling System with Water Cage Chipmunk 


\section{APO Service Building/Target Vault Partial Elevation View}

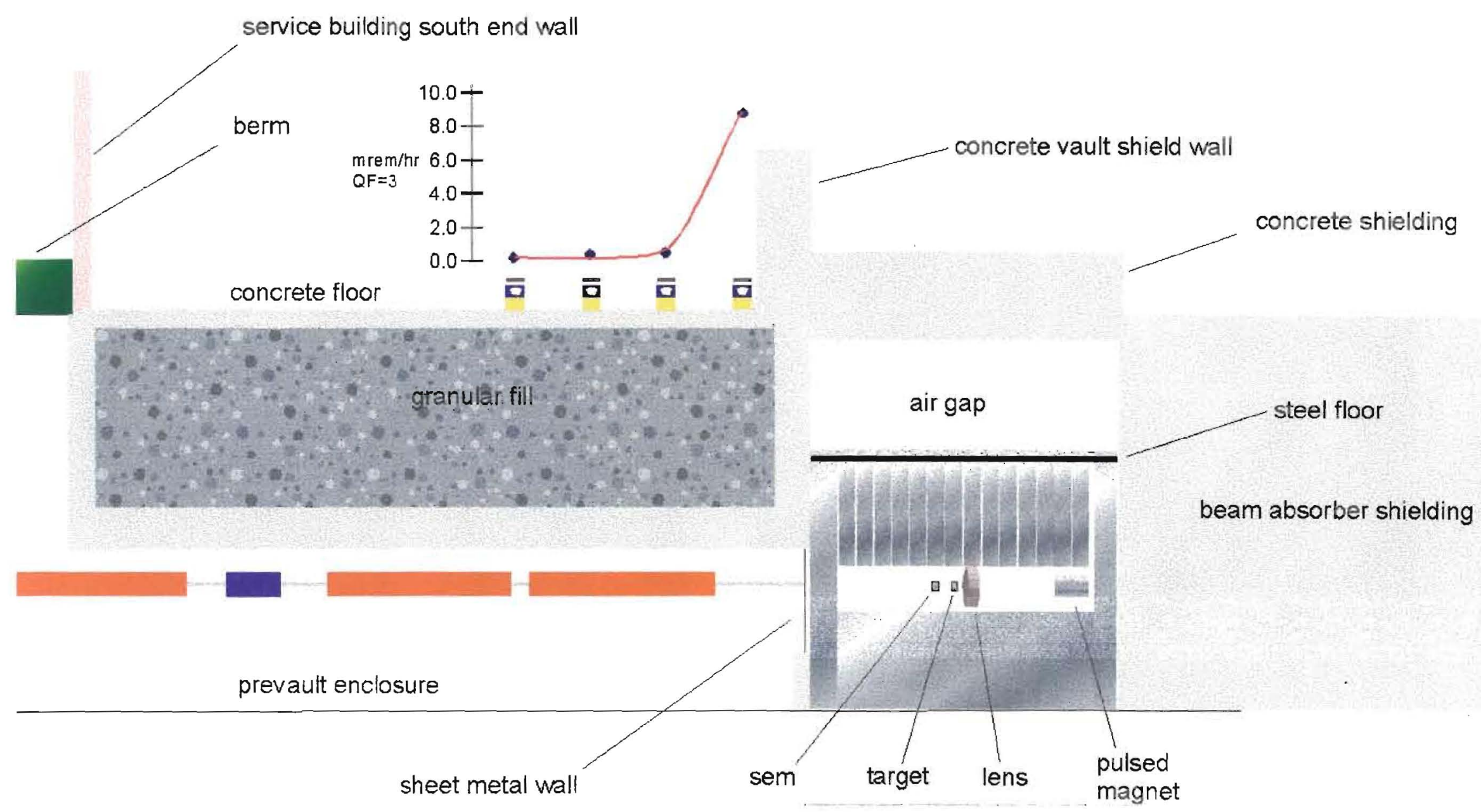

